\section{REFERENCES}

1. Henry BL, Minassian A, Young JW, Paulus MP, Geyer MA, Perry W. Cross-species assessments of motor and exploratory behavior related to bipolar disorder. Neurosci Biobehav Rev. 2010;34:1296-306. https://doi.org/10.1016/j.neubiorev.2010.04.002

2. Teicher MH. Actigraphy and motion analysis: new tools for psychiatry. Harv Rev Psychiatry. 1995;3:18-35.

3. Tecott LH, Nestler EJ. Neurobehavioral assessment in the information age. Nat Neurosci. 2004;7:462-6. https://doi.org/10.1038/nn1225

4. Andreu-Perez J, Poon CC, Merrifield RD, Wong ST, Yang GZ. Big data for health. IEEE J Biomed Health Inform. 2015;19:1193-208. https://doi.org/10.1109/ JBHI.2015.2450362
5. Collier S, Monette P, Hobbs K, Tabasky E, Forester BP, Vahia IV. Mapping movement: applying motion measurement technologies to the psychiatric care of older adults. Curr Psychiatry Rep. 2018;20:64 https://doi.org/10.1007/s11920-0180921-z

6. Shoaib M, Bosch S, Incel OD, Scholten H, Havinga PJ. Complex human activity recognition using smartphone and wrist-worn motion sensors. Sensors. 2016;16:426 https://doi.org/10.3390/s16040426

7. Kumar S, Abowd G, Abraham WT, al'Absi M, Chau DHP, Ertin E, et al. Center of excellence for mobile sensor data-to-knowledge (MD2K). IEEE Pervasive Comput. 2017;16:18-22. https://doi.org/10.1109/MPRV.2017.29

\title{
Treating cue-reactivity with brain stimulation: a new (transdiagnostic) approach
}

\author{
Colleen A. Hanlon ${ }^{1,2,3}$ \\ Neuropsychopharmacology (2019) 44:232-233; https://doi.org/10.1038/s41386-018-0215-5
}

The struggle between internal self-control and external temptation from environmental cues is a tale as old as written history, yet as relevant today as any time in the past. Just as Homer wrote about Odysseus and the seductive Siren Songs (800 B.C.), or Tintorreto painted Adam's temptation in the Garden of Eden (1551 A.D.), twenty-first century depictions of life frequently highlight the struggle to maintain focus despite proverbial "apples" that interrupt our journey. For most individuals, the occasional surrender to a tempting cue will not impair their ability to fulfill daily and longer term responsibilities. For other individuals, however, elevated reactivity to positive or negative cues causes a disabling cascade of events ultimately impeding long-term goals. Elevated cue-reactivity is also a prominent feature of alcohol and substance-use disorder, posttraumatic stress disorder (PTSD), and obsessive behavior disorders, such as eating and gambling.

In these populations, salient cues evoke elevated activity in a consistent network of neural regions: the ventral medial prefrontal cortex (MPFC), anterior cingulate cortex (ACC), and insula. This network may be thought of as a "transdiagnostic neural biomarker" for cue-reactivity. In substance-abuse literature, meta-analyses have demonstrated that these regions are reliably activated by drug cues and may predict relapse [1, 2]. In a recent study by our group, 156 substance dependent individuals performed a drug cue-exposure task tailored to their drug of choice (55 cocaine, 53 alcohol, 48 nicotine) [3]. Multivariate kmeans clustering revealed three distinct clusters of elevated activity when the participants were viewing the drug cues vs. neutral non-drug cues: the MPFC/ACC, the left inferior frontal gyrus/insula, and the right premotor cortex.

From a therapeutic perspective, novel non-invasive brain stimulation treatment protocols are being designed to target the MPFC-ACC-Insula circuit directly [4]. In the cue-reactivity study described above, cortical projection analysis revealed that the frontal pole (FP) was the cortical location closest to the maximal number of significant cue-reactivity clusters. A recent shamcontrolled study in 49 individuals demonstrated that continuous theta burst stimulation (TBS) - a particularly potent and efficient form of transcranial magnetic stimulation (TMS) - directed to the left FP decreases drug cue-reactivity among heavy alcohol users and cocaine users [5]. This protocol also decreases functional connectivity in this MPFC/ACC/Insula network [6].

FP TMS is also being used to improve cue-reactivity in PTSD and obsessive behavioral disorders. Dr Rebecca Price and colleagues at the University of Pittsburg, e.g., are currently evaluating FP TBS, as a tool to decrease compulsive behaviors in obsessive compulsive disorder, many of which are cue-evoked (NCT \#03265015). The use of this MPFC-ACC-Insula network as a framework for modulating cue-reactivity is just beginning. Although there will be several challenges associated with developing TMS strategies to modulate this network (e.g., reaching these deep targets, disease-tailored protocols), the MPFC-ACC-Insula network appears to be a fruitful and transdiagnostic neural biomarker to explore for next generation brain stimulation protocols.

\section{FUNDING}

Dr. Hanlon's research has been funded by the National Institute of Health. Dr. Hanlon receives compensation as a consultant for Brain Research \& Development Services.

\section{ADDITIONAL INFORMATION}

\section{REFERENCES}

1. Janes AC, Pizzagalli DA, Richardt $S$, de BFB, Chuzi S, Pachas $G$, et al. Brain reactivity to smoking cues prior to smoking cessation predicts ability to maintain tobacco abstinence. Biol Psychiatry. 2010;67:722-9.

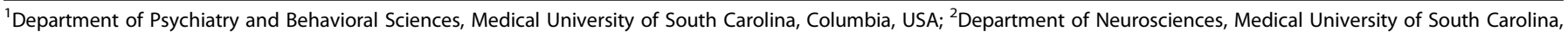
Columbia, USA and ${ }^{3}$ Center for Biomedical Imaging, Medical University of South Carolina, Columbia, USA

Correspondence: Colleen A. Hanlon (hanlon@musc.edu)
}

Published online: 7 October 2018 
2. Seo D, Lacadie CM, Tuit K, Hong KI, Constable RT, Sinha R. Disrupted ventromedial prefrontal function, alcohol craving, and subsequent relapse risk. JAMA Psychiatry. 2013;70:727-39.

3. Hanlon CA, Dowdle LT, Gibson NR, Li X, Hamilton S, Canterberry M, et al. Cortical substrates of cue-reactivity: transdiagnostic relevance of the medial prefrontal cortex. Transl Psychiatry. 2018;8:186.

4. Hanlon CA, Dowdle LT, Henderson JS. Modulating neural circuits with transcranial magnetic stimulation: implications for addiction treatment development. Pharmacol Rev. 2018;70:661-83.
5. Kearney-Ramos TE, Dowdle LT, Lench DH, Mithoefer OJ, Devries WH, George MS, et al. Transdiagnostic effects of ventromedial prefrontal cortex transcranial magnetic stimulation on cue reactivity. Biol Psychiatry Cogn Neurosci Neuroimaging. 2018;3:599-609.

6. Hanlon CA, Dowdle LT, Correia B, Mithoefer O, Kearney-Ramos T, Lench D, et al. Left frontal pole theta burst stimulation decreases orbitofrontal and insula activity in cocaine users and alcohol users. Drug Alcohol Depend. 2017;178:310-7.

\title{
Decoding the role of the microbiome on amygdala function and social behaviour
}

\author{
John F. Cryan $\mathbb{1}^{1,2}$ and Timothy G. Dinan ${ }^{2,3}$ \\ Neuropsychopharmacology (2019) 44:233-234; https://doi.org/10.1038/s41386-018-0233-3
}

We are living in a microbial world with our bodies having as many microbial cells as human cells. Growing evidence implicates these microbes, known collectively as the microbiome, as key regulators of brain function and behaviour [1]. One of the key findings from across many species is that the microbiome affects social behaviour [2]. We have shown that germ-free (GF) mice, which grow up in a sterile environment and thus have no bacteria in or on their bodies, are less sociable than normal mice[2]. Moreover, the amygdala, a brain region important for social behaviour, is particularly sensitive to changes in microbiome composition [3] and GF mice have widespread changes in amygdala neuronal morphology and function [4].

Ongoing research is trying to determine the molecular mechanisms underpinning such effects. Initially, we exploited unbiased genome-wide transcriptional profiling to determine gene expression in the amygdala of male GF mice. We found differential gene expression, exon usage and RNA-editing in GF mice (Fig. 1). We noticed upregulation of several immediate early response genes such as Fos, Fosb, Egr2 or Nr4a1 in association with increased cAMP response element-binding protein (CREB) signalling in GF mice [5]. In addition, we found differential expression and recoding of several genes implicated in a variety of neuronal processes such as neurotransmission, neuronal plasticity, metabolism and morphology. These data strongly suggest altered baseline neuronal activity in the amygdala of GF animals, which may underpin the social deficits. However, what happens under a social stimulus remained known.

To this end we recently described dynamic regulation of several previously undescribed pathways in response to social stimulation. These include regulation of RNA-processing non-coding RNAs that are crucially involved in splicing regulation. Moreover, social stimulus evoked an increase in transcripts of genes involved in neuronal activity, which includes induction of several well established immediate early genes such as Fos or Arc, the MAP-K pathway and neurotrophic signalling via Bdnf. Moreover, we find upregulation of complement components, which have lately been established to be necessary for synaptic rearrangements and plasticity upon neuronal activity [6].

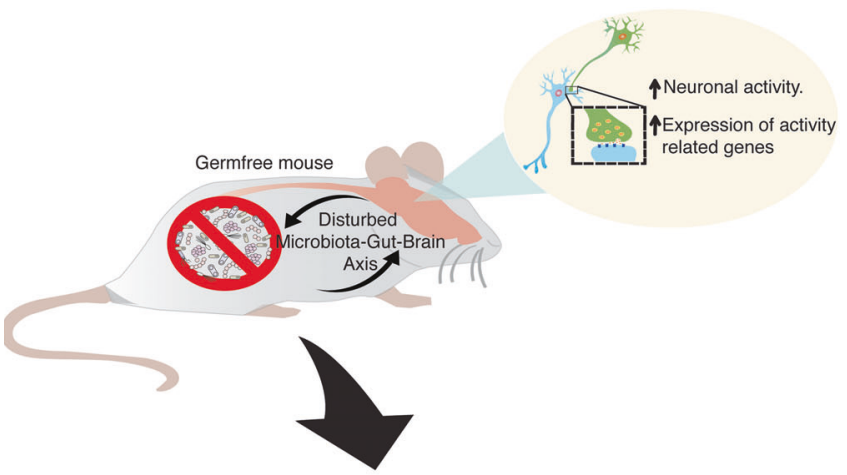

Social interaction behaviour paradigm
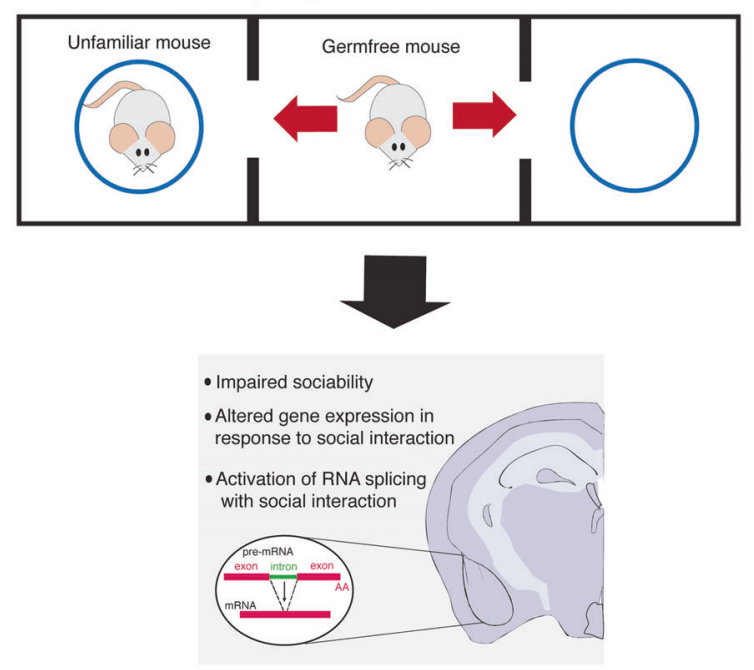

${ }^{1}$ Department of Anatomy and Neuroscience, University College Cork, Cork, Ireland; ${ }^{2}$ APC Microbiome Institute Ireland, University College Cork, Cork, Ireland and ${ }^{3}$ Department of Psychiatry and Neurobehavioral Science, University College Cork, Cork, Ireland

Correspondence: John F. Cryan (j.cryan@ucc.ie)

Received: 14 August 2018 Accepted: 23 September 2018

Published online: 19 October 2018 